\title{
Ascites Worsening Due to a Large Hiatal Hernia Compressing Hepatic Venous Outflow: A Case Report
}

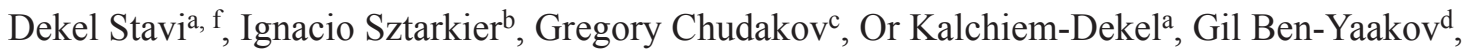 \\ Doron Zilberman ${ }^{\mathrm{a}, \mathrm{e}}$, Emanuel Sikuler ${ }^{\mathrm{a}, \mathrm{e}}$
}

\begin{abstract}
A hiatal hernia causing or aggravating ascites by compressing the hepatic veins has been rarely reported in adults. We report a patient with a known hiatal hernia who was presented with refractory ascites. Echocardiography and CT examination demonstrated a huge hiatal hernia compressing the right atrium and the inferior vena cava. A right-sided heart catheterization demonstrated a significant pressure gradient between the inferior vena cava and the right atrium. Liver biopsy showed a nodular pattern and congestion without fibrosis. Hernioplasty was performed and an improvement in the rate of the accumulation of ascetic fluid was observed. Three months later and during a follow-up period of 3 years, the ascites was mild and there was no need for any therapeutic paracentesis. The outcome indicates that the huge hiatal hernia, by creating hepatic venous outflow obstruction, was the underlying mechanism for ascites aggravation.
\end{abstract}

Keywords: Intractable ascites; Hiatal hernia; Hepatic venous outflow obstruction

\section{Introduction}

There are many causes for ascites. Portal hypertension due to liver cirrhosis is the most common mechanism. Other causes

Manuscript accepted for publication October 18, 2013

a Department of Medicine B, The Soroka Medical Center and Faculty of Health Sciences, Ben-Gurion University of the Negev, Beer-Sheva, Israel

bDepartment of Pathology, The Soroka Medical Center and Faculty of Health Sciences, Ben-Gurion University of the Negev, Beer-Sheva, Israel

cDepartment of Radiology, The Soroka Medical Center and Faculty of Health Sciences, Ben-Gurion University of the Negev, Beer-Sheva, Israel

dInstitute of Gastroenterology, The Soroka Medical Center and Faculty of Health Sciences, Ben-Gurion University of the Negev, Beer-Sheva, Israel

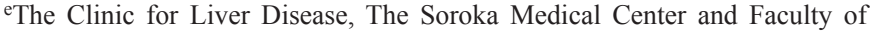
Health Sciences, Ben-Gurion University of the Negev, Beer-Sheva, Israel

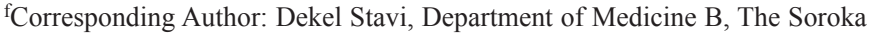
Medical Center, PO Box 151, Beer-Sheva 84101, Israel.

Email: Dekel.stavi@gmail.com

doi: http://dx.doi.org/10.14740/jmc1559w are portal hypertension due to non-cirrhotic etiologies, peritoneal diseases, lymphatic obstruction, hypoalbuminemia and intra-abdominal organ leak (such as pancreatic acites).

Ascites is a common manifestation of hepatic venous outflow obstruction, i.e. Budd-Chiari syndrome [1]. However, ascites due to external compression of the hepatic veins is a rare situation. It has been reported in adults in cases of abdominal trauma, solid tumors, cyst or abscess [2-4].

A hiatal hernia causing ascites by compressing the hepatic veins has been rarely reported in newborns with congenital diaphragmatic pathology $[5,6]$. To the best of our knowledge, it was never reported in adults. We present a case report of an adult patient in whom a huge hiatal hernia contributed to formation of refractory ascites that improved significantly after surgical repair.

\section{Case Report}

A 57-year-old male was referred to the emergency room due to recurrent vomiting that started on the same day. Lately an increasing abdominal swelling was observed. The patient had moderate mental retardation and was a resident of a nursing home. His medical history included chronic constipation and a hiatal hernia. His regular medications were clothiapine, valproic acid and promethazine.

Few days previous to his admission, he was examined in the emergency room due to abdominal swelling and was diagnosed as suffering from an inguinal hernia. At that time his chest and abdominal X-rays showed bilateral pleural effusion, with small bilateral atelectasis and a large hiatal hernia without signs of gastrointestinal obstruction or perforation. An abdominal X-ray following oral contrast material administration revealed normal passage. The inguinal hernia was reduced and the patient was discharged.

On the day of his admission, the abdominal swelling increased and he started to vomit. On his arrival to the emergency room, his blood pressure was $145 / 70 \mathrm{~mm} \mathrm{Hg}$, heart rate was 120 beats/min, body temperature was $37.2^{\circ} \mathrm{C}$ and the respiratory rate was $20 / \mathrm{min}$. On physical examination, he was dyspneic, the jugular veins were mildly distended and the heart sounds were normal without murmurs or a friction rub. The lung examination was normal. The abdomen was swollen without guarding sings. An averted umbilicus was observed 


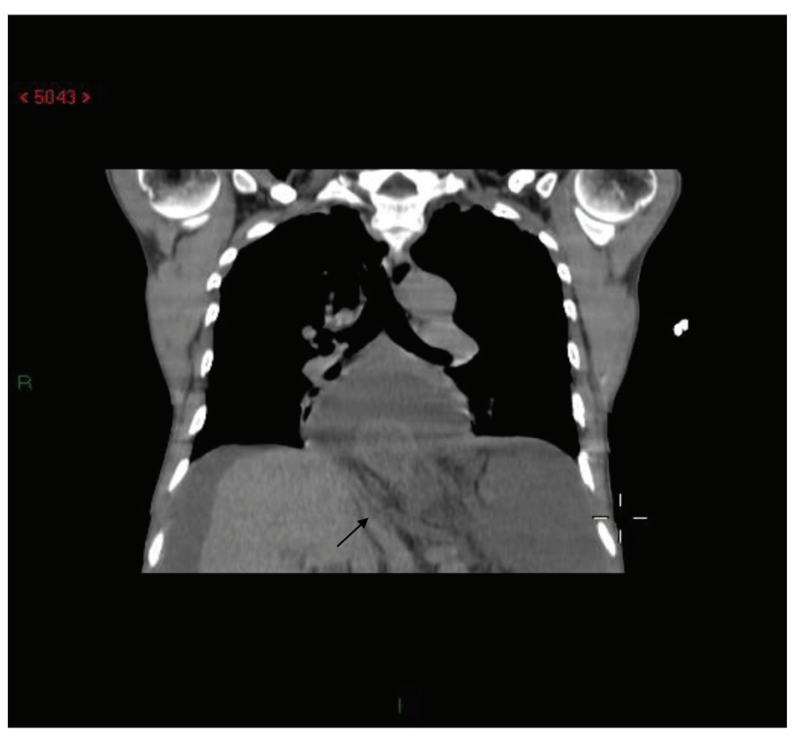

Figure 1. Multiplanar reconstruction (MPR) - coronal view without IV contrast media showing huge hiatal hernia compressing the inferior vena cava in proximity to the confluence of hepatic veins.

and shifting dullness was found. There was no leg edema and the peripheral arterial pulses were normal.

ECG showed low voltage sinus tachycardia of 120 beats/ min, the axis was undetermined and otherwise there were no QRS or ST-T abnormalities. Blood electrolytes were normal, creatinine level was $0.78 \mathrm{mg} / \mathrm{dL}$, total bilirubin was $0.7 \mathrm{mg} / \mathrm{dL}$, and aspartate aminotransferase (AST) and alanine transaminase (ALT) were 59 and $23 \mathrm{IU} / \mathrm{L}$ respectively. Alkaline phosphatase, gamma-glutamyl transpeptidase (GGT) and lactate dehydrogenase (LDH) levels were 89, 128 and 813 IU/L respectively. Pancreatic enzymes were normal, and albumin and globulin levels were 3.4 and $4.4 \mathrm{~g} / \mathrm{dL}$ respectively. The hemoglobin level was $14.5 \mathrm{~g} / \mathrm{dL}$, and the total white blood cell (WBC) and platelets count were 10,800 and $428,000 / \mu \mathrm{L}$ respectively.

His chest and abdominal X-rays were as described above.

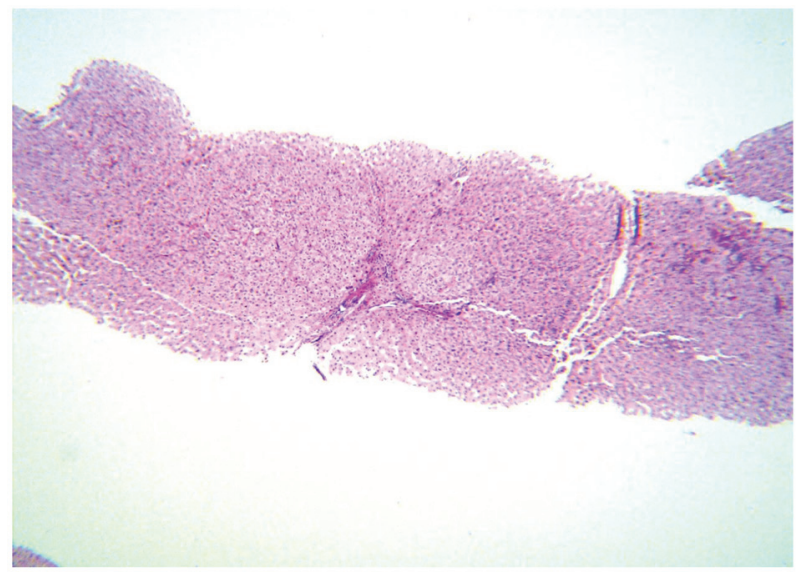

Figure 2. Low power view - nodular configuration of the liver tissue without fibrosis.

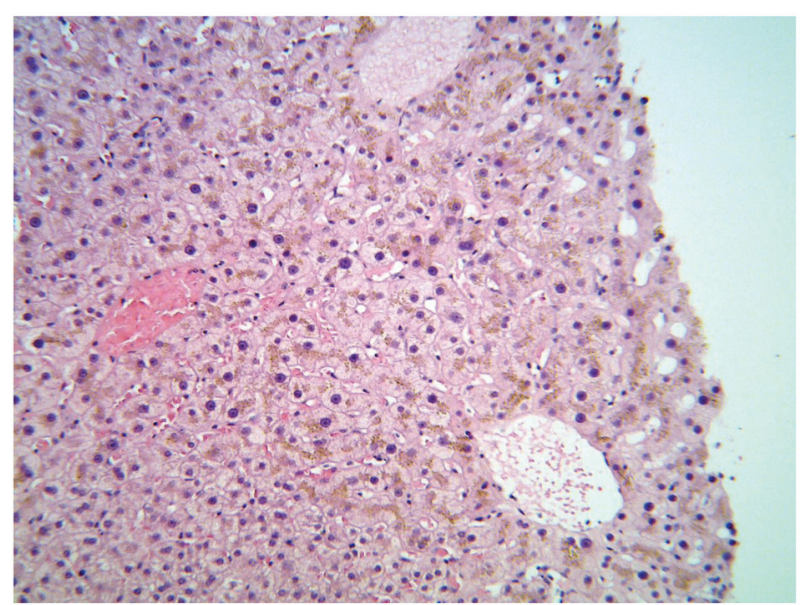

Figure 3. Low power view - congestive central veins and some congested sinusoids and prominent lipofuscin pigmentation seen in the hepatocytes.

The patient was hospitalized for further investigation. Due to dyspnea and large abdominal swelling, a large volume abdominal paracentesis, draining $7 \mathrm{~L}$ of ascetic fluid was conducted. The total cell count of the ascetic fluid was 460 cells $/ \mu \mathrm{L}$ with $33 \%$ neutrophils. The albumin level of the peritoneal fluid was $1.8 \mathrm{~g} / \mathrm{dL}$, hence the serum ascites albumin gradient (SAAG) was $1.6 \mathrm{~g} / \mathrm{dL}$. Fluid cultures were negative, and the cytological examination was normal.

Anti-hepatitis $\mathrm{C}$ virus (HCV) and hepatitis B surface antigen (HBsAg) were negative, anti-nuclear antibody (ANA), rheumatoid factor (RF), and anti-neutrophil cytoplasmic antibody (ANCA) were negative, and compliments levels were normal. The alphafetoprotein level was $14.1 \mathrm{ng} / \mathrm{mL}$, and protein electroforesis showed a polyclonal hypergammaglobulinemia.

Echocardiography showed a normal left ventricular systolic function, without any valvular abnormalities. The right ventricle was normal in size and function. An extra cardiac lesion compressing the atria was identified.

A chest and abdominal contrast enhanced CT showed a

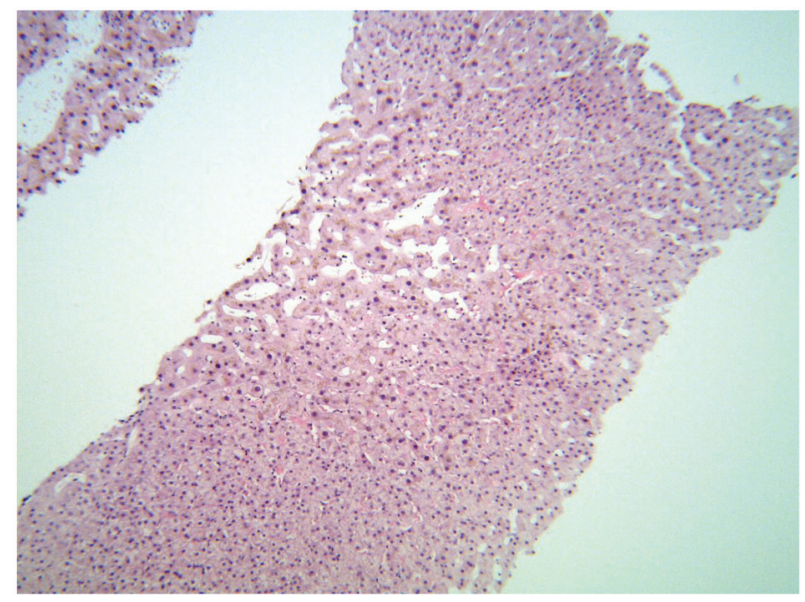

Figure 4. Dilated sinusoidal spaces are present. 
huge hiatal hernia, compressing the thoracic inferior vena cava (Fig. 1). The hepatic veins were patent, the liver and spleen were normal in texture and size, and the abdominal cavity contained a large amount of free fluid. A gastroscopy revealed a large hiatal hernia, with mild hypertensive gastropathy. Esophageal varices were not observed.

The wedge and free hepatic venous pressures were 17 and $13 \mathrm{~mm} \mathrm{Hg}$ respectively, thus the hepatic venous pressure gradient was $4 \mathrm{~mm} \mathrm{Hg}$. A needle liver biopsy revealed a nodular pattern with venous congestion without significant fibrosis (Figs. 2-4).

A right-sided heart catheterization was conducted. The mean right atrial was $17 \mathrm{~mm} \mathrm{Hg}$ and a pressure gradient between the inferior vena cava and the right atrium was found. The other measurements were within normal limits and no signs of pericardial constriction were observed.

Following abdominal paracentesis, a temporary improvement in the respiratory distress was observed. However, accumulation of ascites was rapid despite salt restriction and diuretic therapy and repeated procedures of large volume paracentesis were indicated.

At that point we concluded that obstruction of hepatic venous outflow, due to compression of the supra hepatic inferior vena cava by the huge hiatal hernia, contributed to the formation of the intractable ascites. Thus, a laparoscopic hernioplasty was performed.

During the first 2 months after surgery, despite salt restriction and diuretic therapy, the patient needed three more procedures of large volume paracentesis. On the third postoperative month, ascites accumulation was dramatically decreased without any need for further therapeutic paracentesis.

We followed the patient up to 3 years later. There were no signs of ascites on physical examination. Nevertheless, ascetic fluid was still detectable by abdominal ultrasound.

\section{Discussion}

The presented patient had ascites with a high serum - ascites albumin gradient, indicating that portal hypertension was the underlying etiology. A large ascites without the presence of esophageal varices implied that the mechanism was post sinusoidal obstruction.

The major cause of intrahepatic post sinusoidal obstruction is the hepatic "sinusoidal obstructive syndrome". Nodular regenerative hyperplasia may rarely present as ascites [7]. The histological findings were compatible with nodular regenerative hyperplasia. Nevertheless, this condition cannot explain the congestion of the jugular vein. Moreover, the rapid accumulation of ascetic fluid, recent to his admission, suggested another etiology. The cardiac echocardiogram and catheterization did not support a myocardial, valvular or pericardial pathology. Normally, there is no measurable pressure gradient between the inferior vena cava and the right atrium. The finding of an external pressure on the right atrium combined with a pressure gradient between the inferior vena cava and the right atrium led to the conclusion that the findings are due to external compression by the huge hiatal hernia. Therefore, hernioplasty was performed.

The hiatal hernia, most probably, was not the cause for the ascites formation but it was the cause of worsening ascites. This conclusion is supported by the fact that the hiatal hernia was known for many years, and that the ascites formation reduced dramatically following hernioplasty.

After the operation, the improvement had not evolved immediately but rather after few weeks. This sequence of events is not surprising. First, the patient had probably an underlying intra-hepatic post sinusoidal liver pathology that was not cured by the hernioplasty. Second, as after treating Budd-Chiari syndrome the improvement can be delayed due to structural changes of the liver tissue secondary to the extrahepatic venous obstruction. Nevertheless, in contrast to the preoperative refractory massive ascites, the patient remained with mild ascites detectable only by ultrasonography.

Portal hypertension caused by external compression of the inferior vena cava was reported in cases of trauma, malignancy or cysts. Hiatal hernia is a common medical condition. So is ascites. The combination of the two is known as well. Nevertheless, ascites worsened by a hiatal hernia was never reported in humans according to our best knowledge. Although rare, this condition should be considered in the differential diagnosis of massive ascites and hiatal hernia.

\section{Disclosure}

All the authors declare that there is no conflict of interest and there is no funding support.

\section{References}

1. Valla D, Benhamou JP. Obstruction of the hepatic veins or suprahepatic inferior vena cava. Dig Dis. 1996;14(2):99118.

2. Akbulut S, Yilmaz M, Kahraman A, Yilmaz S. Budd-Chiari syndrome due to giant hydatid cyst: a case report and brief literature review. J Infect Dev Ctries. 2013;7(6):489493.

3. Menon KV, Shah V, Kamath PS. The Budd-Chiari syndrome. N Engl J Med. 2004;350(6):578-585.

4. Kaifi JT, Kavuturu S, Judelson D, Staveley-O'Carroll KF. Budd-Chiari syndrome and post-traumatic diaphragmatic hernia. J Thorac Cardiovasc Surg. 2012;143(4):e21-22.

5. Giacoia GP. Right-sided diaphragmatic hernia associated with superior vena cava syndrome. Am J Perinatol. 1994;11(2):129-131.

6. Gilsanz V, Emons D, Hansmann M, Meradji M, Donaldson JS, Omenaca F, Quero J, et al. Hydrothorax, ascites, and right diaphragmatic hernia. Radiology. 1986;158(1):243246.

7. Wanless IR. Micronodular transformation (nodular regenerative hyperplasia) of the liver: a report of 64 cases among 2,500 autopsies and a new classification of benign hepatocellular nodules. Hepatology. 1990;11(5):787-797. 